\title{
Transition Metals for the Synthesis of Glycopolymers and Glycopolypeptides
}

\author{
Maidul Islam, ${ }^{[a]}$ Ashif Y. Shaikh, ${ }^{[b]}$ and Srinivas Hotha*[a]
}

Abstract: Glycopolymers and glycopolypeptides are an important class of molecules, which can self-assemble to various interesting biohybrid materials. It is envisaged that the glycans impart good immunological response, and the aliphatic or polypeptide backbone can give tertiary structure for the resulting glycopolymers. The major bottleneck in the synthesis of glycopolymers or glycopolypeptides is the access to suitable building blocks and polymerization methods. This review describes methods that have recently been explored for the successful synthesis of many useful glycomonomers that could be polymerized to afford glycopolymers and/or glycopolypeptides.

Keywords: glycopeptides · polymerization $\cdot$ synthetic methods $\cdot$ transition metals

\section{Introduction}

Cellular information starts with the replication of DNA, followed by transcription of the information to the RNA, which gets subsequently translated into a protein that further undergoes post-translational modifications, among which glycosidation is the most complicated. ${ }^{[1]}$ Proteins function as enzymes to catalyze reactions to give a variety of polysaccharides, polyketides, and terpenes, which are proven to be of immense benefit to mankind (Figure 1). ${ }^{[1 \mathrm{a}]}$ Nature exploits extraordinary mechanical properties of abundantly available polysaccharides, such as starch, cellulose, and chitin, for its benefit. Glycans exist as linear or branched forms, and in addition, they can differ by stereochemical linkage at the anomeric or $C$ -

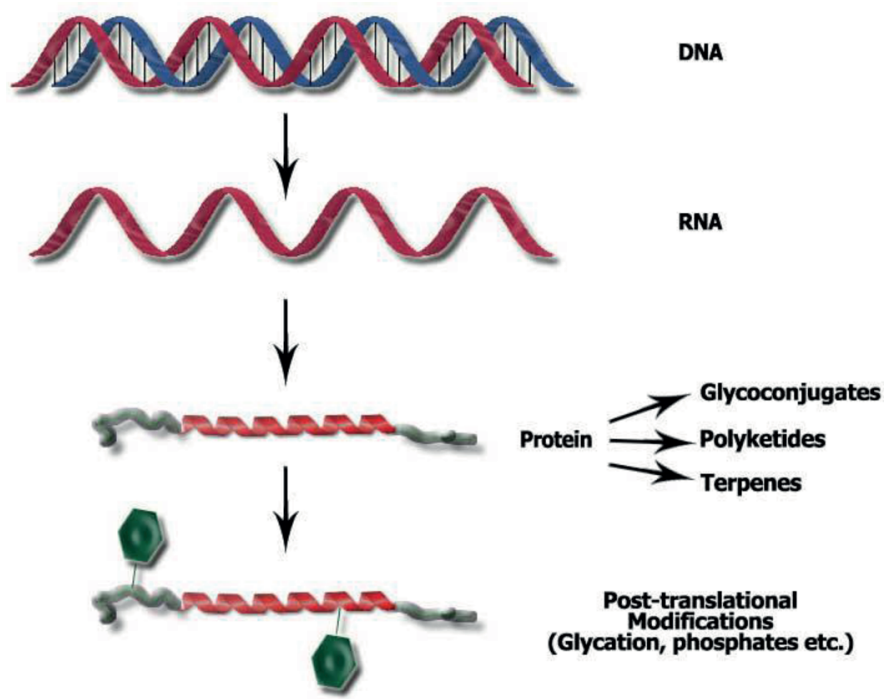

Figure 1. Expanded central dogma of molecular biology.
1 position to generate exceptional diversity. ${ }^{[2]}$ The gigantic structural diversity and information stored in the glycome represents the next challenge of biology, and its potential is still underestimated. ${ }^{[3]}$ Bioinformatic studies estimate that half of the human proteins undergo post-translational glycosylation to form glycoproteins, which are often present on the cell surface. ${ }^{[1,3]}$

Cell surface glycans are often attached to either a lipid or a protein, and therefore, are known as glycolipids or glycoproteins, and are broadly referred to as glycoconjugates. ${ }^{[4]}$ Cell surface bound glycoconjugates are found to play pivotal roles in many intracellular and extracellular signal transduction events (Figure 2). ${ }^{[1 \mathrm{~b}]}$ For instance, glycoproteins are demonstrated to be involved in cell-cell communication, cell-cell adhesion, fertilization, viral entry into the cell, bacterial infection, and cell-cell recognition, and hence, they are recognized to have potential applications in therapeutics, diagnostics, and vaccines. ${ }^{[1 \mathrm{~b}]}$ In addition, many biohybrid polymers are currently investigated as novel smart materials, since they are envisaged to have solubility, processability, and scalability, along with much desired chirality and cellular recognition. ${ }^{[5]}$ Isolation of glycoproteins from nature is a daunting task,

[a] M. Islam, S. Hotha

Department of Chemistry

Indian Institute of Science Education and Research

Pune 411008 (India)

e-mail: s.hotha@iiserpune.ac.in

[b] A. Y. Shaikh

Institute of Materials Science and Nanotechnology National Nanotechnology Research Center (UNAM) Bilkent University

Ankara 06800 (Turkey) 
Maidul Islam received his B.Sc. (2009) and M.Sc. (2011) in chemistry from University of Calcutta, in India. At present, he is a Ph.D. student in the research group of Prof Srinivas Hotha at IISER Pune. Stereoselective glycosylations and synthesis of novel glycoconjugates are the focus of his research.

Ashif Shaikh is currently working as a postdoctoral fellow at National Nanotechnology Research Center (UNAM), Bilkent University, Ankara (Turkey), funded by the TUBITAK-2216 fellowship programme. His postdoctoral research is focused on synthesis of glycopeptide nanofibers for biomedical application. He received his Ph.D. degree in chemistry from the University of Pune (renamed as Savitribai Phule Pune University), for research work carried out with Prof. Srinivas Hotha at
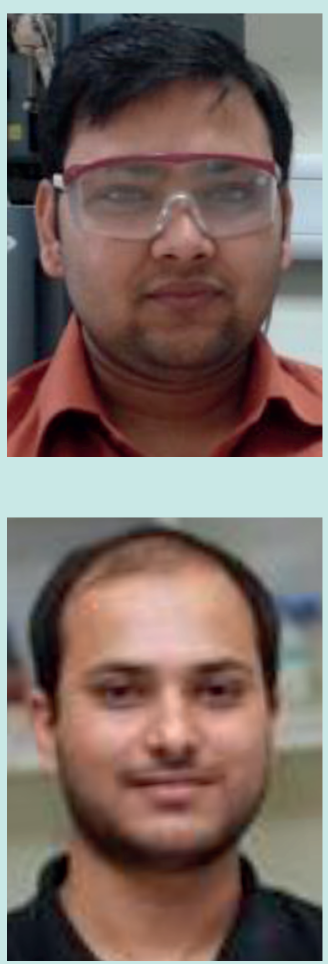
the CSIR-National Chemical Laboratory, Pune (India), in 2013. During his Ph.D., he developed a new high yielding glycosidation method for attachment of amino acids to carbohydrates, using gold catalysts and transformation of the thus synthesized glycoaminoacid derivatives were converted to Glyco-NCAs for glycopolypeptide synthesis. His current research is focused on integrating carbohydrates with peptides for efficient delivery systems and tissue engineering scaffolds.

Srinivas Hotha graduated with an M.Sc. degree from the School of Chemistry, University of Hyderabad, and subsequently with an M.Tech. in Biochemical Engineering from the Institute of Technology, Banaras Hindu University, Varanasi. Hotha earned his Ph.D. from Osmania University, Hyderabad for research work carried out at the Indian Institute of Chemical Technology, Hyderabad and National Chemical Laboratory, Pune in 2001. He was the Charles H. Revson Fellow in the

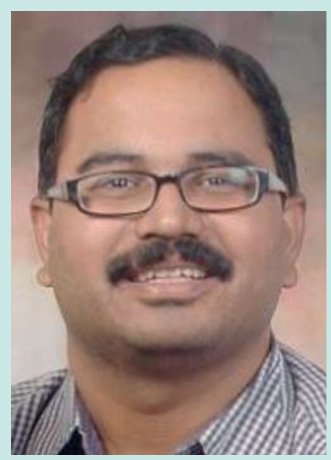

Laboratory of Chemistry and Cell Biology at the Rockefeller University, USA from 2001 to 2003. Hotha moved to the National Chemical Laboratory in 2003 to further his research, and subsequently joined IISER Pune as an Associate Professor - Chemistry (2010). Hotha received the Young Scientist (equivalent to early career in the Western world) award from leading academies of India. He has been honoured with a CDRI Research Award in Chemical Sciences (2014), CRSI Bronze Medal (2014), and Hotha was the recipient of the prestigious Swarnalayanti Fellowship in Chemical Sciences from the Department of Science \& Technology, New Delhi in 2010. His current research is focused on the synthesis of glycopolymers and oligofuranosides using gold catalysis.

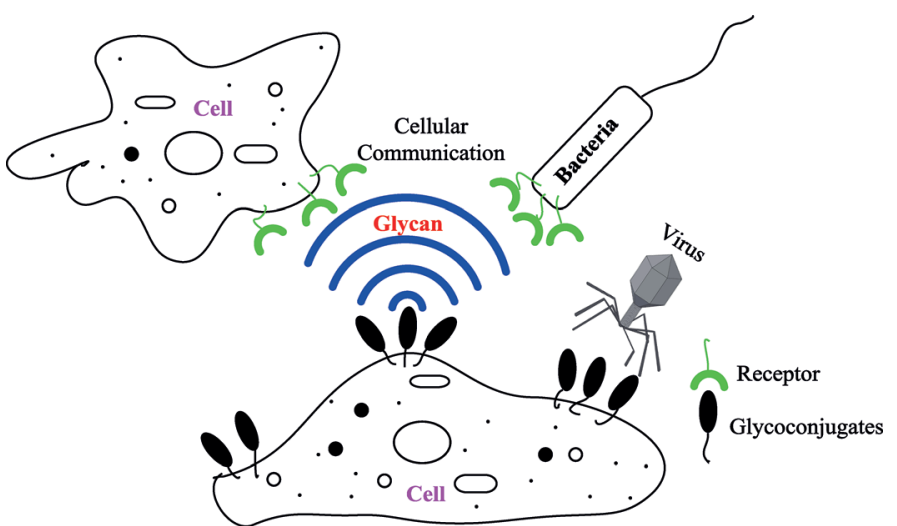

Figure 2. Significance of cell surface glycoconjugates.

as they exist in micro-heterogeneous forms, and there is no universal protocol for the expression of homogeneous glycoproteins yet. ${ }^{[6]}$ In this regard, synthetic glycopolymers will be of great significance in understanding various cellular recognition events for an eventual modulation.

Methods currently available for the synthesis of glycopolymers can be classified into three broad categories (Figure 3$){ }^{[7]}$ First, a monomer with a unique functional group that will enable post-polymerization attachment of glycans can be subjected to the polymerization conditions. Subsequently, the unique functional group can be utilized for orthogonally grafting glycans to convert them into glycopolymers (Method 1, Figure 3). For example, Haddleton and coworkers have shown the utility of an azidealkyne 1,3-dipolar cycloaddition reaction, which is more popular among "click" reactions; Gress, Völkel, and Schlaad ${ }^{[8]}$ have utilized the thiol-ene "click" reaction for the synthesis of glycopolymers; and Perrier's group ${ }^{[9]}$ have used it for highly branched glycopolymers. Apart from the addition of a thiol-bearing carbohydrate to alkene and alkyne, it can be also coupled to pentafluorostyrene moieties, substituting the para-positioned fluorine ${ }^{[10]}$ and pentafluorophenyl acrylate activated ester to make scaffolds for tissue engineering. ${ }^{[1]}$ As the saccharide pendants are attached after the polymer synthesis, one can easily diversify the glycan without worrying about the polymer synthesis; but, the characterization of the glycopolymer should be carried out. The post-polymer grafting method will pose an ambiguity in the total number of glycans and their distribution along the polymeric backbone (Figure 3). ${ }^{[12]}$

The second, and more widely used, method dwells upon the ligation of a glycan to a suitably end-functionalized polymer to obtain glycopolymers (Method 2, Figure 3). Native chemical ligation has been used for the attachment of glycans to a model peptide. ${ }^{[13]}$ This approach may not be really suitable for studying the dendritic or glycocluster effect. ${ }^{[14]}$

The third, and more advantageous, method of glycopolymer syntheses is the polymerization of a carbohydrate 


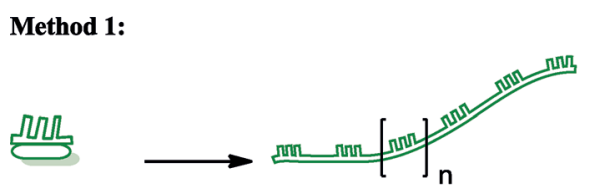

Method 2:
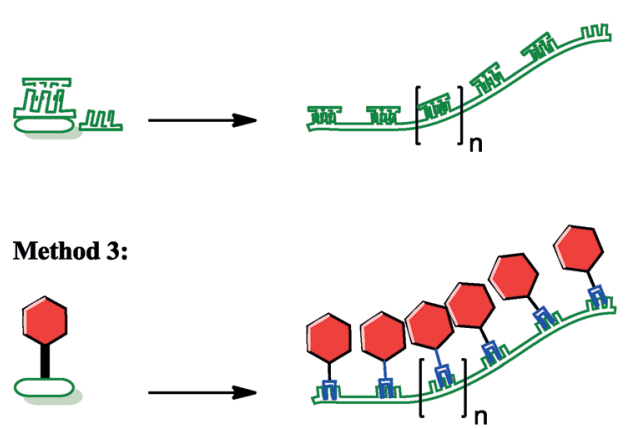
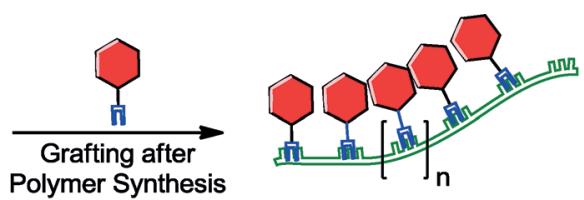

Polymer Synthesis

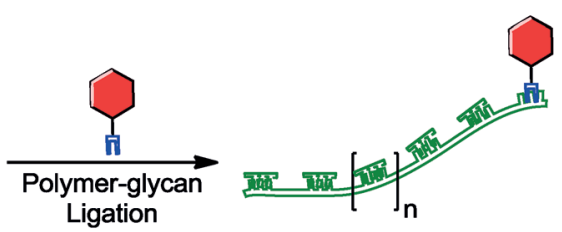

Ligation

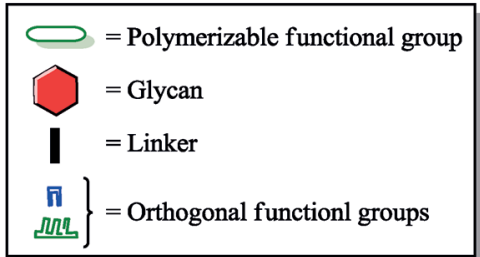

Figure 3. Popular strategies of glycopolymer syntheses.

residue, with a pendant polymerizable functional group, under suitable conditions, to afford good glycan density, as well as periodicity (Method 3, Figure 3). For example, glycopolymers can be synthesized by a free radical polymerization of acryloyl and acrylamido glycosides, ${ }^{[15 a]}$ and glycopolypeptides can be obtained by the ring-opening polymerization $^{[15 \mathrm{~b}-\mathrm{e}]}$ of $N$-carboxyanhydride $(\mathrm{NCA})^{[16]}$ containing sugar-amino acid hybrid molecules.

\section{Results and Discussion}

Glycopolymers can be obtained by applying modern methodologies developed by the contemporary synthetic organic chemistry community. For instance, glycopolymers were synthesized by the Kent-pioneered native chemical ligation (NCL), wherein a thioester endcapped polymer was treated with another polymer containing cysteine, so that a conjugate of the polymer could be obtained. ${ }^{[13]}$ Also, any two polymers can be ligated by using the dichlorotetrazine as the linker, if the polymers are endcapped with suitable functional groups. ${ }^{[17]}$

Copper assisted azide-alkyne 1,3-dipolar cycloadditions are also reported for the synthesis of conjugating two independently synthesized polymers, wherein one of the polymers bears the alkyne and the other polymer has an azide capping. ${ }^{[18]}$ However, the key in all these approaches is the easy access to monomer-glycosides. Traditional methods for the synthesis of these sugar-monomer glycosides suffer from one or more limitations, such as harsh reaction conditions, long and tedious work-up, and laborious purification of highly reactive sugar monomers. In the era of green chemistry, synthesis by the use of a cat- alytic amount of reagents, procedures that require no or minimal purification, and are atom economical are in great demand. ${ }^{[19]}$ In addition, the glycosidation procedure should not yield a diastereomeric mixture of glycosides, as their separation is challenging, due to the presence of highly reactive and polymerizable appendages. ${ }^{[12]}$

Glycoproteins can be synthesized by attaching a glycan to the already synthesized peptide/protein, using various bioconjugation techniques. Danishefsky's group reported the synthesis of $\mathrm{N}$-glycoprotein by utilizing the glycal assembly method for the synthesis of carbohydrate epitopes, solid-phase peptide synthesis for polypeptide synthesis, and final ligation under NCL (Scheme 1). ${ }^{[20]}$ Alternatively, a glycan and a polypeptide can be joined using the $\mathrm{Cu}$-mediated azide-alkyne 1,3-dipolar cycloaddition reaction to obtain the already synthesized glycan and polypeptide. ${ }^{[18]}$ One can also synthesize glycopolymers by choosing appropriate building blocks, while conducting solid-phase peptide synthesis (Figure 4).

In all these cases, access to amino acid glycoconjugates is the key, and development of an efficient synthetic methodology would greatly benefit the entire glycobiology and materials community immensely.

\subsection{Mercury Cyanide for Glycopolymer Synthesis}

Rüde prepared the first $O$-glycosylated $N$-carboxy anhydride (NCA) by the use of modified Koenig-Knorr glycosidation conditions ${ }^{[21]}$ to study immunological properties of glycopolypeptides. ${ }^{[16]}$ After several years, Okada's group synthesized glycoamino acid building blocks by Rüde's method and studied synthesis of glycopolypeptides. $^{[22]}$ 
Glycal assembly method



Solid Phase Peptide Synthesis (SPPS)

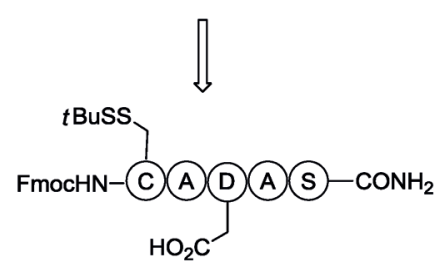

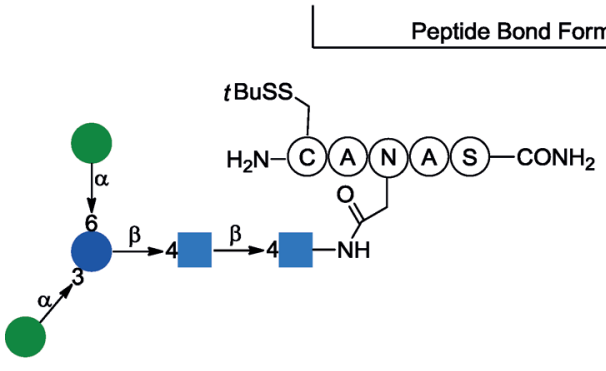

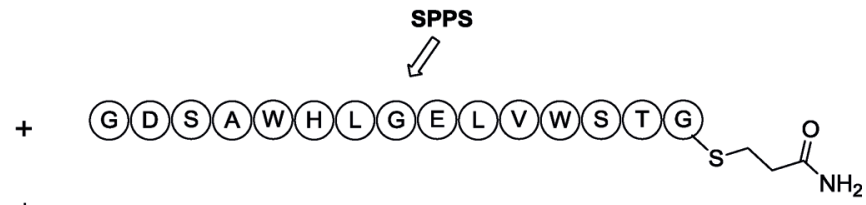

Native Chemical Ligation

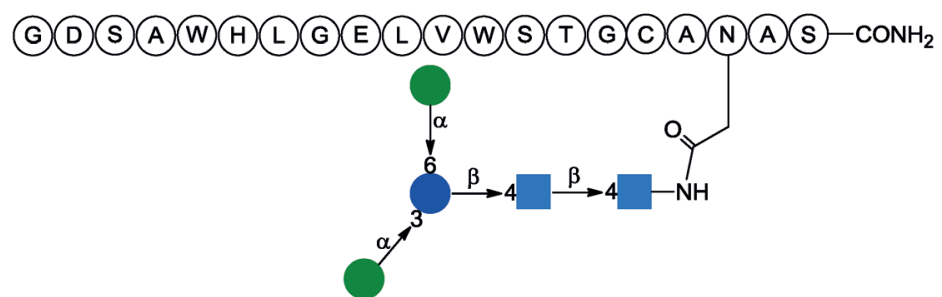

Scheme 1. Schematic of the fully synthetic $N$-glycoprotein by solid-phase peptide synthesis (SPPS) and native chemical ligation.

Confirmation of the living nature of primary amine-initiated polymerization was shown by synthesizing an ABtype block copolymer, with sequential addition of monomers (Scheme 2). ${ }^{[22]}$ Nevertheless, successful synthesis of glycopolypeptides through NCA was a significant advancement, though the required monomer synthesis conditions are environmentally benign and low yielding.

\subsection{Cobalt, Nickel and Ruthenium for Glycopolypeptide and Glycopolymer Synthesis}

High molecular weight, well-defined polypeptides were obtained by NCA living polymerization using transition metal derived complexes. Deming and coworkers obtained controlled NCA polymerization, $\mathrm{Ni}(\mathrm{COD})$ (bipy), and found it to be the best catalytic system to obtain glycopolypeptides. ${ }^{[21]}$

Further, they explored the application of $\mathrm{Co}\left(\mathrm{PMe}_{3}\right)_{4}$ catalysts for synthesis of glycopolypeptides; further, redox-triggered helix to coil transition onto the secondary conformation was shown, without loss of water solubility (Scheme 3). ${ }^{[23]}$ The power of ruthenium catalysis was demonstrated through ring-opening metathesis polymerization (ROMP) of norbornenyl appended glucosamine to afford glycopolymers with various densities, and its lectin binding ability was studied (Scheme 4). ${ }^{[24]}$

\subsection{Gold and Silver Catalysis for Glycopolymer Synthesis}

In this context, a gold-catalyzed glycosidation repertoire was found to be advantageous, as: (1) the reaction is high yielding; (2) the reaction requires a catalytic quantity of reagents; (3) the glycosyl donor is stable and easy to access; (4) no work up is necessary; and (5) several functional groups, such as alkenes, esters, and ethers, are tolerated under the $\mathrm{Au}(\mathrm{III})$-catalysis conditions. ${ }^{[25 \mathrm{a}]}$ Of the two variations of the gold-catalyzed glycosidation, exploration of the synthesis of glycopolymers by the use of propargyl 1,2-orthoesters was preferable, as highly diastereoselective glycosidation occurs at the room temperature. ${ }^{[25 \mathrm{~b}]}$ Many different glycopolymers were synthesized in the 1960 s by controlled radical polymerization of vinyl monomers, and later by ring-opening metathesis polymerization of norbornenes, which have carbon backbones. ${ }^{[26]} \mathrm{Li}$ and Yu reported an unprecedented glycosylation-initiated cationic ring-opening polymerization (CROP) of tetrahydrofuran, employing ortho-hexynylbenzoates as glycosyl donors, in the presence of a catalytic amount of $\mathrm{Au}(\mathrm{I})$ catalyst (Scheme 5). ${ }^{[27]}$

However, the first polymerizable glycomonomer synthesis, under gold(III)-catalyzed glycosidation conditions, was reported in 2011, by the use of propargyl 1,2-orthoesters as glycosyl donors (Scheme 5). ${ }^{[28]}$ It was observed that propargyl 1,2-orthoesters undergo glycosida- 
Incorporation of one sugar residue into a peptide backbone

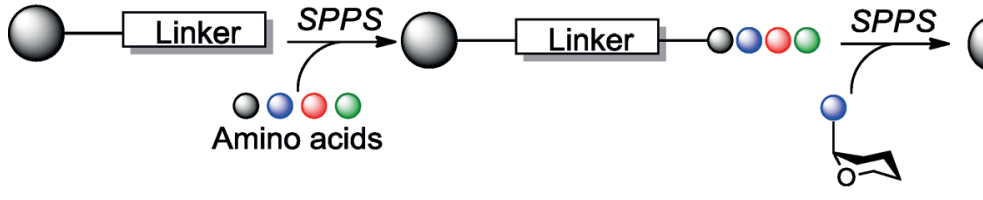

Glyco amino acid
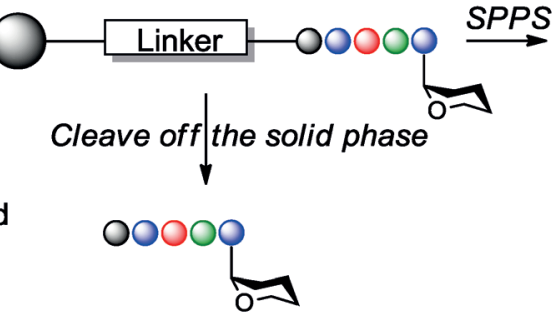

'Click' chemistry for ligating a glycan and a polymer
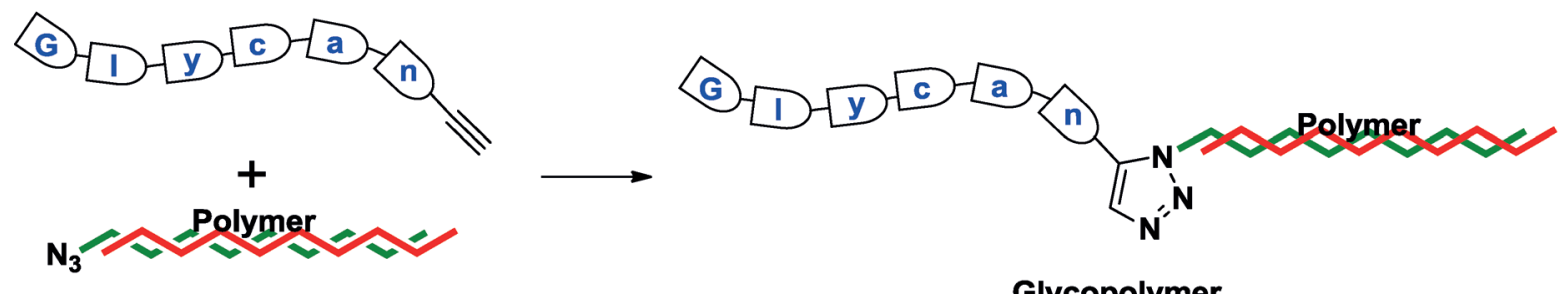

Glycopolymer

Native Chemical Ligation when glycan and polymer are known

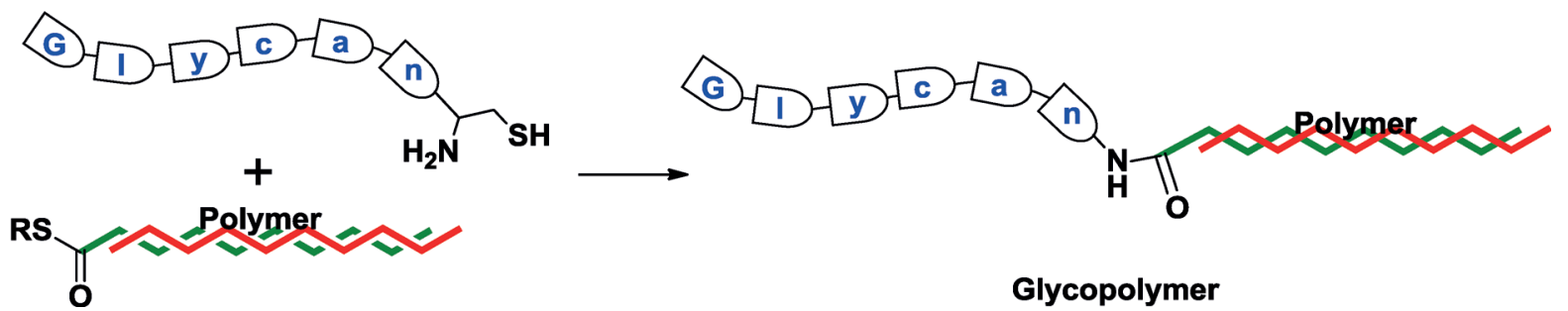

Figure 4. Methods for the synthesis of hybrid glycopolymers with the fixed glycan and polymer positions.

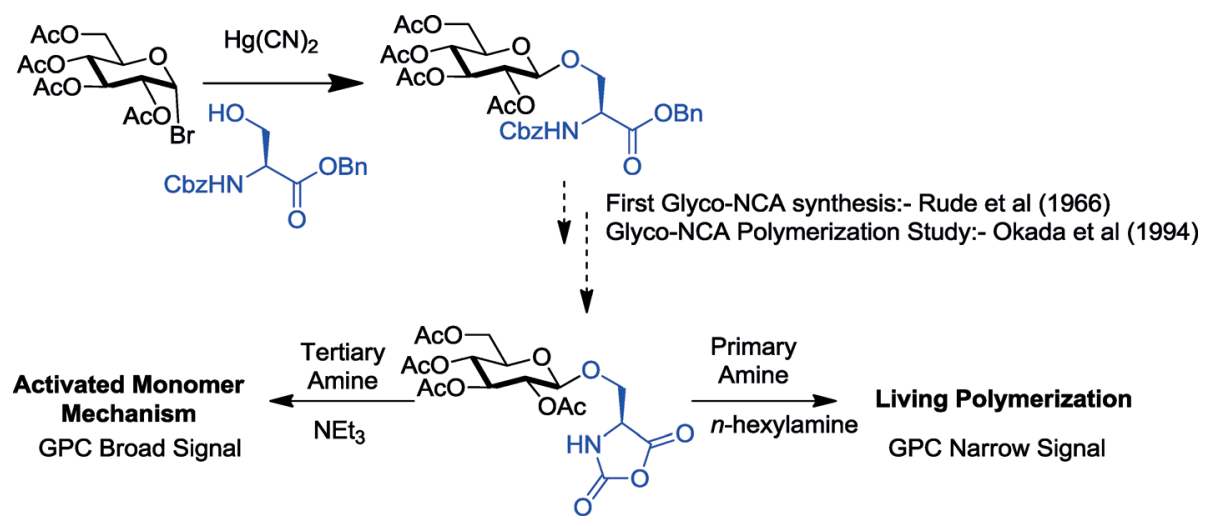

Scheme 2. Synthesis of glyco- $N$-carboxy anhydride (NCA).

tion with hydroxyethyl acrylates, giving very good yield of the glyco-acrylate monomers in the presence of $7 \mathrm{~mol} \%$ $\mathrm{AuBr}_{3}$ in anhydrous $\mathrm{CH}_{2} \mathrm{Cl}_{2}$. The reaction conditions do not require aqueous-phase work up, and hence, simple filtration through a pad of Celite was sufficient to obtain glycomonomers that were suitable for free radical polymerization. ${ }^{[12]}$
Many glyco-acrylate monomers were synthesized using this protocol; however, the synthesis of carbohydrate-acrylamide monomers required a two-step procedure, as the above reaction conditions afforded the trans-orthoester, which was subsequently treated with another Lewis acid to obtain carbohydrate-acrylamide monomers. ${ }^{[12]}$ Furthermore, thus prepared sugar monomers were subjected to 




Scheme 3. Synthesis of glycopolypeptides by cobalt catalysis.

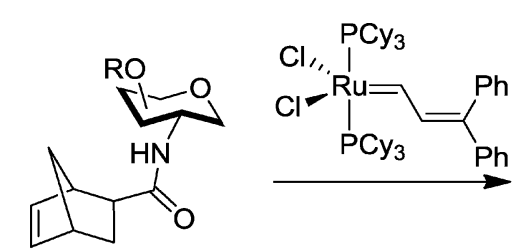

$\mathrm{R}=-\mathrm{H},-\mathrm{Ac},-\mathrm{Bn},-\mathrm{Tr}$

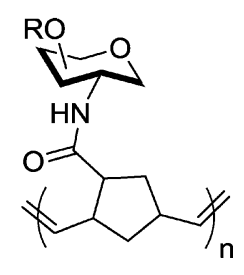

Scheme 4. Synthesis of glycopolymers by ROMP.

free radical polymerization to obtain glycopolymers with a good polydispersity index. ${ }^{[12]}$ Additionally, glycomonomers were shown to undergo the thiol-ene "click" reaction to give yet another dimension to the thus prepared glyco-acrylate/acrylamide monomers.

Glycopolypeptides have received special attention in the recent past, as they have the intriguing ability to fold into higher order structures, due to several noncovalent interactions between amide units. ${ }^{[21]}$ Inherent biocompatibility and biodegradability of glycosylated polypeptides, and their inherent influence in anti-freezing, proliferation of cells, and inflammatory reactions, enticed various practitioners to dwell upon the development of methods for the synthesis of glycopolypeptides. Glycopolypeptides mimic the chemical structure of glycoproteins, and hence serve as models for: (1) understanding physicochemical or biological properties of natural glycoproteins; (2) elucidating the fundamental structure-property relationships; and (3) the eventual design of smart hybrid materials. ${ }^{[7]}$

Synthetic glycopeptides have been known for a long time; however, large scale synthesis of them is still a formidable task, and knowledge about their self-assembled structures for the design of smart functional materials is still in its infancy. Several synthetic methodologies are developed for the synthesis of glycopolypeptides, either to perfectly reproduce the chemical structure and/or function of the naturally occurring glycoprotein, or to synthesize biohybrid glycoproteins that can mimic the function of glycoproteins. The total synthesis of erythropoietin is a good example for the amalgamation of methods existing in carbohydrate, peptide, and bioconjugate chemistries, in a multi-disciplinary effort. ${ }^{[29]}$ In this synthetic endeavour, Merrifield-pioneered solid peptide synthesis was used for synthesizing fragments of glycopeptides that were effectively subjected to native chemical ligation to obtain fully synthetic erythropoietin. Methods available in the literature now enable researchers to synthesize unnatural amino acids and new glycosidic linkages. However, the key challenge in the synthesis of glycopolypeptides is still the making of the bond between the amino acid and the glycan in a diastereoselective fashion. For a long time, amino acid glycoconjugates were synthesized under Koenig-Knorr glycosidation conditions; the reaction yields were moderate to poor and the demands extensive, including laborious purification. The gold-catalyzed glycosidation for the amino acid glycoconjugate was found to be beneficial for the synthesis of amino acid glycoconjugates (Scheme 6). ${ }^{[28]}$

The reaction was observed to be highly diastereoselective, with minimal requirement for purification, and high yielding, with Fmoc- and Cbz- protecting groups. However, when the protection on the amino acid was changed to the tert-butoxycarbonyl ( $t$-Boc), two products were noticed, which were identified as a trans-orthoester product and glycosyl carbamate. ${ }^{[28]}$ Formation of glycosyl carbamate was attributed to the acidic reaction conditions that facilitated the cleavage of the tert-butyl group, producing a highly reactive carbamic acid. Usually, carbamic acids liberate $\mathrm{CO}_{2}$, affording free amines; however, under the reaction conditions, the carbamic acid was observed to 
Gold(I)-catalysis for neoglycopolymers

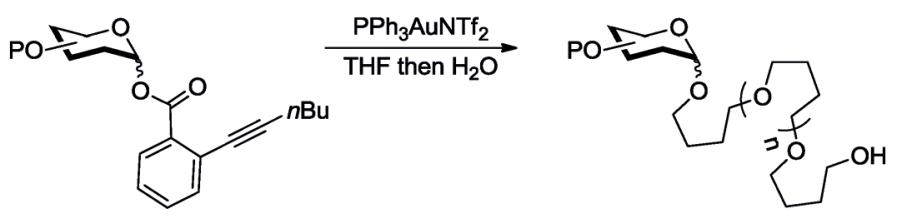

Gold(III)-catalysis for polymerizable glycomonomers
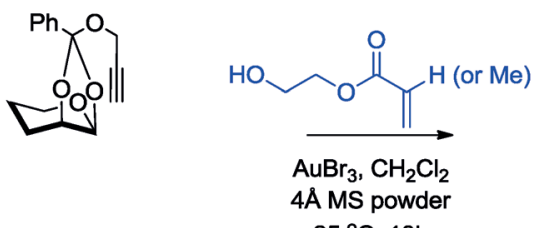

$25^{\circ} \mathrm{C}, 10 \mathrm{~h}$

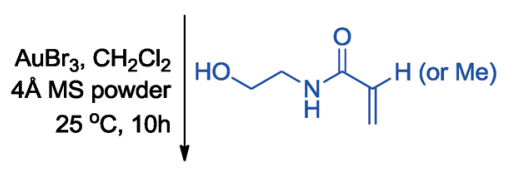

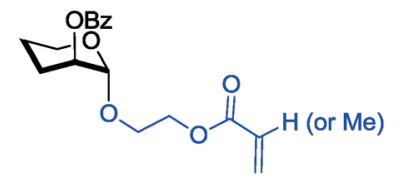

Glycosidation with $7 \mathrm{~mol} \%$ of $\mathrm{Au}(\mathrm{III})$ salt

No work-up necessary

Monomers suitable for polymerization

Upto $78 \%$ yield<smiles>C=CC(=O)NCCOC1(c2ccccc2)OC2CCCC(O2)O1</smiles>

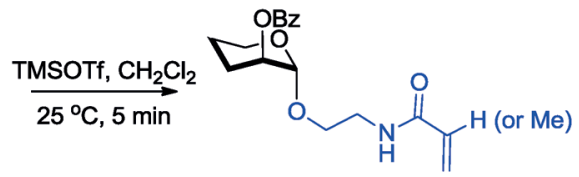

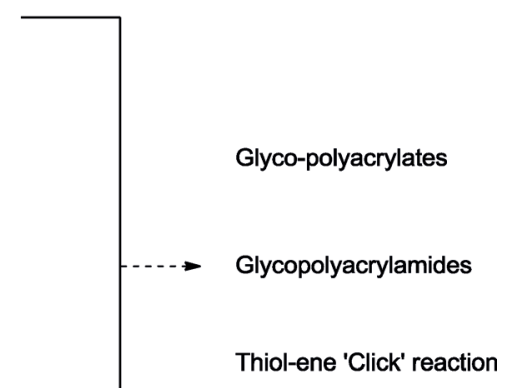

Scheme 5. Gold catalysis for the synthesis of glycopolymers.

Scheme 5. Gold catalysis for the synthesis of glycopolymers.

attack the oxocarbenium ion generated in the reaction medium from the propargyl 1,2-orthoesters. The reaction was further optimized to afford exclusively glycosyl carbamates, if the gold-catalyzed glycosidation was conducted with Boc-protected phenyl alanine and $\mathrm{HAuCl}_{4}$ (Scheme 6). ${ }^{[28]}$

Further, the protocol was also successfully applied for the synthesis of end-functionalized and amphiphilic diblock copolymers. The block copolymerizations proceeded very well to give end-functionalized and amphiphilic glycopolymers; further, azide present at the end of the glycopolypeptide was clicked, under CuAAC conditions, to obtain fluorescently labelled glycopolypeptides. Saponification of esters from the carbohydrate residues of the synthesized glycopolypeptides enabled water-soluble glycopolypeptides, whose lectin binding studies were investigated for mannose specific Concanavalin A lectin binding. ${ }^{[30 \mathrm{~d}]}$ More recently, the conjugation between glycopolypeptides and hydrophobic dendrons was effected by the CuAAC reaction, and the thus obtained molecules were observed to undergo self-assembly, affording multiple topologies in the form of organogels, nanorods, and micellar aggregates, depending on the polypeptide chain length and dendron generation. ${ }^{[30 \mathrm{a}]}$ Self-assembled glyco- polypeptide nanostructures might be useful for biomedical applications.

In another effort, glycopolypeptides synthesized through gold-catalyzed glycosidation were conjugated, under CuAAC conditions, to silk fibroin, the natural fibrous protein created by the Bombyx mori silk worm. ${ }^{[30]}$ Thus formed silk fibroin glycopolypeptide hybrid material showed very high affinity towards Concanavalin A, and preliminary experiments showed that the material is biocompatible; thus, it is anticipated to have potential use in tissue engineering. ${ }^{[30]}$ Gold catalysis was also beneficially utilized for the synthesis of 6-deoxy-6-azido glycopolypeptides by the NCA polymerization. ${ }^{[30 \mathrm{c}]}$

A different approach for the synthesis of carbohydrate appended glycopolymers was described by Deming and Kramer under silver catalysis conditions. ${ }^{[181]}$ Methionine was found to undergo a chemoselective, and highly efficient, alkylation reaction to form stable sulfonium derivatives (Scheme 7).

This is the first post-polymerization modification of methionine polypeptides by glycans using the transition metal catalyst, $\mathrm{AgBF}_{4}$, to get glycosylated polypeptides. Unactivated carbohydrate halide attachment to methionine proceeded upon addition of silver tetrafluoroborate 
Gold(III)-catalysis for Amino acid Glycoconjugates

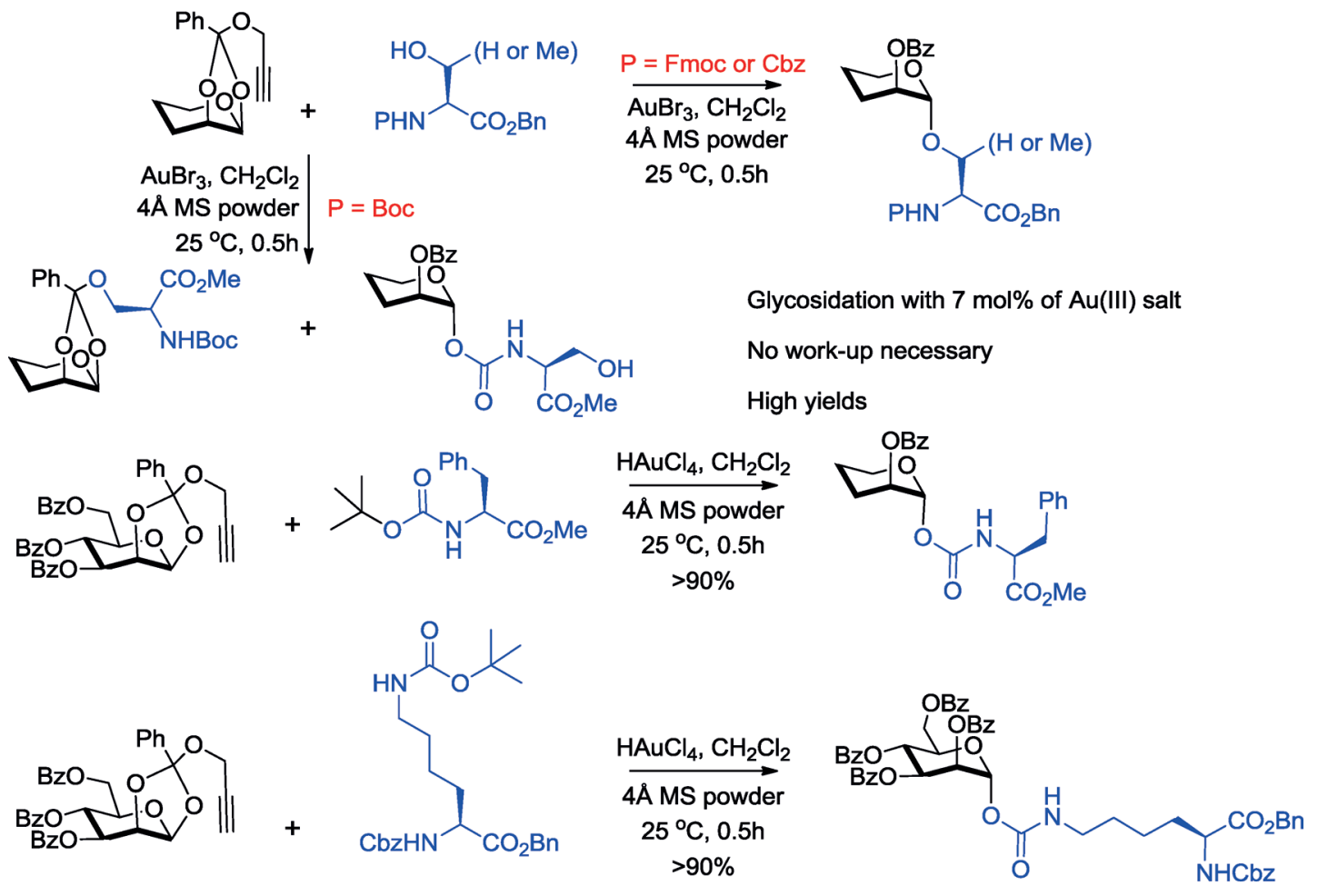

Scheme 6. Synthesis of glycoamino acid building blocks by gold(III) catalysis.
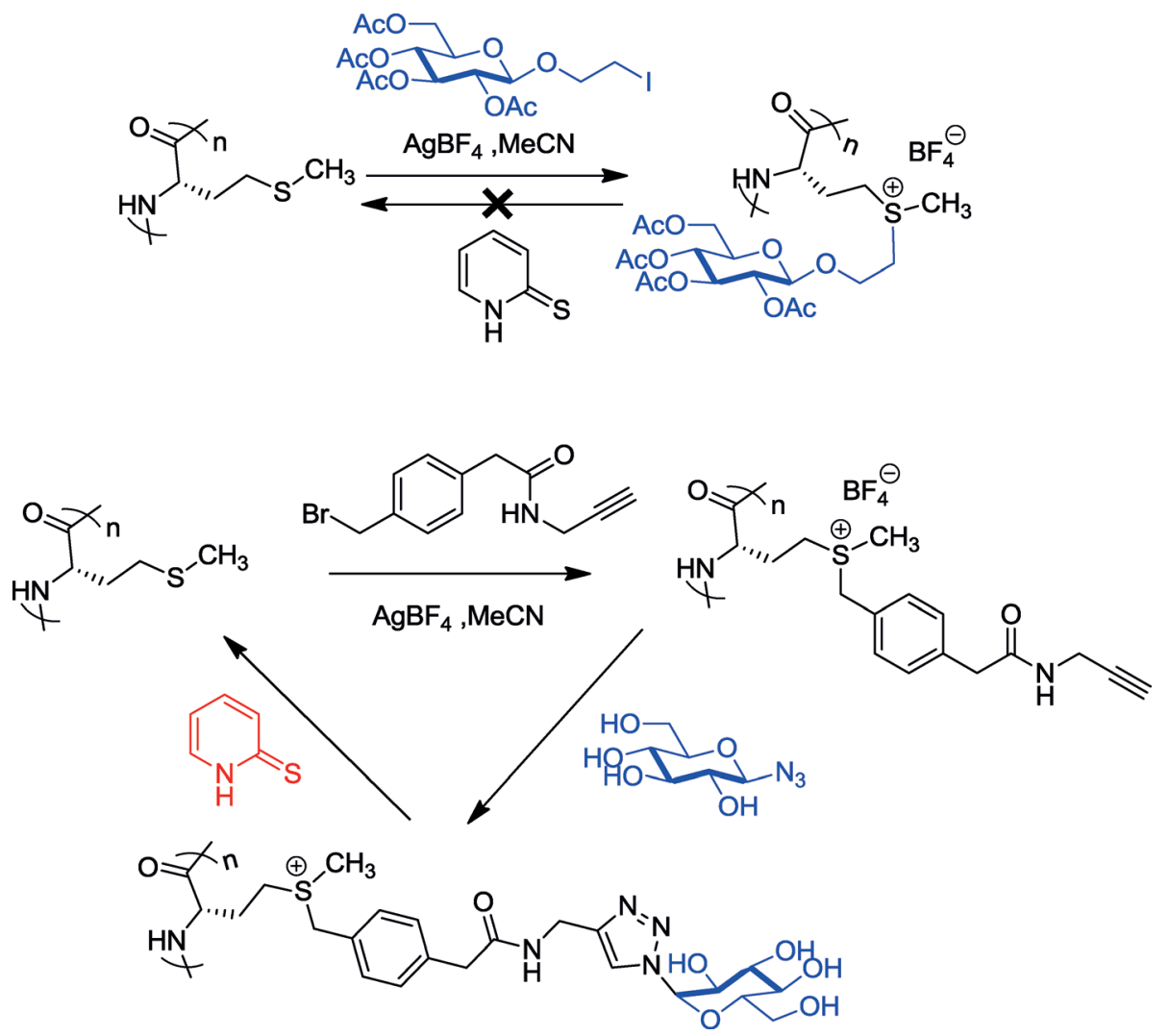

Scheme 7. Silver catalysis for the glycopolymers synthesis. 
to give high molecular weight, well-defined, and fully glycosylated polypeptides. Dynamic control over the modification of polypeptides with glycans was also shown by Deming and Kramer, using a specific alkylating agent via reversible methionine sulfonium salt formation (Scheme 7).

\section{Outlook}

The development of novel synthetic techniques, combining salient features of amino acid, carbohydrate, and polymer chemistries, have enabled preparation of a large number of new biohybrid glycopolymers. The gold-catalysis for glycoconjugates was observed to be superior to existing methods for synthesizing glycomonomers. Easy access to glycopolypeptides would spur more activity from polymer and material chemists to dwell upon synthesizing smart materials, which might open fantastic opportunities in glycomics research to understand the properties of natural glycoproteins. Easy access to biohybrid glycopolymers would spur more activity towards understanding self-assembling properties for eventual applications in medicine and materials.

\section{References}

[1] a) D. V. Vranken, G. Weiss, Introduction to Bioorganic Chemistry and Chemical Biology, Garland Science, New York, 2013, pp. 1-27; b) R. A. Dwek, Chem. Rev. 1996, 96, $683-720$.

[2] P. H. Seeberger, D. B. Werz, Nature 2007, 446, 1046-1051.

[3] H. Rüdiger, H.-J. Gabius, in The Sugar Code (Ed.: H.-J. Gabius), Wiley-VCH, Weinheim, 2009, pp. 3-14.

[4] X. Zhu, R. R. Schmidt, Angew. Chem. Int. Ed. 2009, 48, $1900-1934$

[5] J. R. Kramer, T. J. Deming, Polym. Chem. 2014, 5, 671-682.

[6] D. P. Gamblin, E. M. Scalan, B. G. Davis, Chem. Rev. 2009, 109, 131-163.

[7] a) C. Bonduelle, S. Lecommandoux, Biomacromolecules 2013, 14, 2973-2983; b) K.-S. Krannig, H. Schlaad, Soft Matter 2014, 10, 4228-4235; c) C. Schatz, S. Lecommandoux, Macromol. Rapid Commun. 2010, 31, 1664-1684.

[8] A. Gress, A. Völkel, H. Schlaad, Macromolecules 2007, 40, $7928-7933$

[9] M. Semsarilar, V. Ladmiral, S. Perrier, Macromolecules 2010, 43, 1438-1443.

[10] C. R. Becer, K. Babiuch, D. Pilz, S. Hornig, T. Heinze, M. Gottschaldt, U.S. Schubert, Macromolecules 2009, 42, 2387-2394.

[11] A. S. Hayward, A. M. Eissa, D. J. Maltman, N. Sano, S. A. Przyborski, N. R. Cameron, Biomacromolecules 2013, 14, 4271-4277.

[12] S. A. Thadke, M. Kar, S. S. Gupta, S. Hotha, Carbohydr. Res. 2011, 346, 1511-1518.

[13] a) T. Buskas, P. Thompson, G.-J. Boons, Chem. Commun. 2009, 5335-5349; b) R. J. Payne, C.-H. Wong, Chem. Commun. 2010, 46, 21-43; c) J. Garner, K. A. Jolliffe, M. M. Harding, R. J. Payne, Chem. Commun. 2009, 6925-
6927; d) S. W. A. Reulen, W. W. T. Brusselaars, S. Langereis, W. J. M. Mulder, M. Breurken, M. Merkx, Bioconjugate Chem. 2007, 18, 590-596; e) S. S. Panda, C. D. Hall, A. A. Oliferenko, A. R. Katrizky, Acc. Chem. Res. 2014, 47, $1076-$ 1087; f) C. S. Bennett, C.- H. Wong, Chem. Soc. Rev. 2007, 36, 1227-1238; g) M. R. Pratt, C. R. Bertozzi, Chem. Soc. Rev. 2005, 34, 58-68; h) A. Brik, Y. Y. Yang, S. Ficht, C. H. Wong, J. Am. Chem. Soc. 2006, 128, 5626-5627; i) A. Brik, S. Ficht, Y.-Y. Yang, C.-H. Wong, J. Am. Chem. Soc. 2006, 128, 15026-15033; j) Y. Shin, K. A. Wilnans, B. J. Backes, S. B. H. Kent, J. A. Ellman, C. R. Bertozzi, J. Am. Chem. Soc. 1999, 121, 11684-11689; k) S. Ingale, T. Buskas, G.-J. Boons, Org. Lett. 2006, 8, 5785-5788; 1) D. J. Lee, K. Mandal, P. W. R. Harris, M. A. Brimble, S. B. H. Kent, Org. Lett. 2009, 11, 5270-5273.

[14] J. J. Lundquist, E. J. Toone, Chem. Rev. 2002, 102, 555-578.

[15] a) A. Ghadban, L. Albertin, Polymer 2013, 5, 431-526; b) M. Armes, R. Narain, Biomaterials 2011, 32, 5276-5290; c) K. Kobayashi, H. Sumitomo, Y. Inai, Polym. J. 1985, 17, 565-575; d) A. Narumi, T. Matsuda, H. Kaga, T. Seto, T. Kakuchi, Polymer 2002, 43, 4835-4840; e) C. Fleming, A. Maldjian, D. Da Costa, A. K. Rullay, D. M. Haddleton, J. St. John, P. Penny, R. C. Noble, N. R. Cameron, Nat. Chem. Biol. 2005, 1, 270-274.

[16] E. Rüde, O. Westphal, E. Hurwitz, M. Sela, Immunochemistry 1966, 3, 137-151.

[17] C. F. Hansell; P. Espeel, M. M. Stamenović, I. A. Barker, A. P. Dove, F. E. Du Prez, R. K. O'Reilly, J. Am. Chem. Soc. 2011, 133, 13828-13831.

[18] a) A. Engler, H.-I. Lee, P. Hammond, Angew. Chem. Int. Ed. 2009, 48, 9334-9338; b) C. Xiao, C. Zhao, P. He, Z. Tang, X. Chen, X. Jing, Macromol. Rapid Commun. 2010, 31, 991-997; c) T. Borase, T. Ninjbadgar, A. Kapetanakis, S. Roche, R. O'Connor, C. Kerskens, A. Heise, D. F. Brougham, Angew. Chem. Int. Ed. 2013, 52, 3164-3167; d) H. Tang, D. Zhang, Biomacromolecules 2010, 11, 1585-1592; e) J. Huang, G. Habraken, F. Audouin, A. Heise, Macromolecules 2010, 43, 6050-6057; f) J. Huang, C. Bonduelle, J. Thévenot, S. Lecommandoux, A. Heise, J. Am. Chem. Soc. 2012, 134, 119-122; g) A. J. Rhodes, T. J. Deming, ACS Macro Lett. 2013, 2, 351-354; i) J. Sun, H. Schlaad, Macromolecules 2010, 43, 4445-4448; j) K. Krannig, H. Schlaad, J. Am. Chem. Soc. 2012, 134, 18542-18545; k) K. Krannig, J. Huang, A. Heise, H. Schlaad, Polym. Chem. 2013, 4, 39813986; 1) J. R. Kramer, T. J. Deming, Biomacromolecules 2012, 13, 1719-1723; m) C. Schatz, S. Louguet, J.-F. Le Meins, S. Lecommandoux, Angew. Chem. Int. Ed. 2009, 48, 2572-2575.

[19] P. J. Walsh, H. M. Li, C. A. de Parrodi, Chem. Rev. 2007, $107,2503-2545$.

[20] a) J. Zhu, Q. Wan, G. Ragupathi, C. M. George, P. O. Livingston, S. J. Danishefsky, J. Am. Chem. Soc. 2009, 131, 4151-4158; b) J. Zhu, J. D. Warren, S. J. Danishefsky, Expert Rev. Vaccines 2009, 8, 1399-1413.

[21] a) T. J. Deming, Nature 1997, 390, 386-389; b) A. C. Farthing, J. Chem. Soc. 1950, 3213-3217; c) M. Yu, A. P. Nowak, D. J. Pochan, T. J. Deming, J. Am. Chem. Soc. 1999, 121, 12210-12211.

[22] a) K. Aoi, K. Tsutsumiuchi, M. Okada, Macromolecules 1994, 27, 875-877; b) K. Aoi, K. Itoh, M. Okada, Macromolecules 1995, 28, 5391-5393; c) K. Aoi, K. Tsutsumiuchi, E. Aoki, M. Okada, Macromolecules 1996, 29, 4456-4458; d) K. Tsutsumiuchi, K. Aoi, M. Okada, Macromolecules 1997, 30, 4013-4017. 
[23] a) J. R. Kramer, T. J. Deming, J. Am. Chem. Soc. 2010, 132, 15068-15071; b) J. R. Kramer, T. J. Deming, J. Am. Chem. Soc. 2012, 134, 4112-4115.

[24] a) L. E. Strong, L. L. Kiessling, J. Am. Chem. Soc. 1999, 121, 6193-6196; b) C. W. Cairo, J. E. Gestwicki, M. Kanai, L. L. Kiessling, J. Am. Chem. Soc. 2002, 124, 1615-1619; c) M. Kanai, K. H. Mortell, L. L. Kiessling, J. Am. Chem. Soc. 1997, 119, 9931-9932.

[25] a) S. Hotha, S. Kashyap, J. Am. Chem. Soc. 2006, 128, 96209621; b) G. Sureshkumar, S. Hotha, Tetrahedron Lett. 2007, 48, 6564-6568.

[26] S. R. S. Ting, G. Chen, M. H. Stenzel, Polym. Chem. 2010, 1, 1392-1412.

[27] Y. Li, B. Yu, Chem. Commun. 2010, 46, 6060-6062.

[28] A. Y. Shaikh, G. Sureshkumar, D. Pati, S. S. Gupta, S. Hotha, Org. Biomol. Chem. 2011, 9, 5951-5959.
[29] P. Wang, S. Dong, J.-H. Shieh, E. Peguero, R. Hendrickson, M. A. S. Moore, S. J. Danishefsky, Science 2013, 342, 13571360.

[30] a) D. Pati, N. Kalva, S. Das, G. Kumaraswamy, S. S. Gupta, A. V. Ambade, J. Am. Chem. Soc. 2012, 134, 7796-7802; b) S. Das, D. Pati, N. Tiwari, A. Nisal, S. Sen Gupta, Biomacromolecules 2012, 13, 3695-3702; c) A. Y. Shaikh, S. Das, D. Pati, V. Dhaware, S. S. Gupta, S. Hotha, Biomacromolecules 2014, 15, 3679-3686; d) D. Pati, A. Y. Shaikh, S. Das, P. K. Nareddy, M. J. Swamy, S. Hotha, S. S. Gupta, Biomacromolecules 2012, 13, 1287-1295.

Received: December 21, 2014

Accepted: February 8, 2015

Published online: March 30, 2015 